\title{
Morphing Aircraft: The Need for a New Design Philosophy
}

\author{
Rafic Ajaj ${ }^{1}$ \& Andy Keane ${ }^{4}$ \\ University of Southampton, Southampton, UK \\ Christopher Beaverstock ${ }^{2}$ \& Michael Friswell ${ }^{3}$ \\ Swansea University, Swansea, UK \\ Daniel Inman ${ }^{5}$ \\ University of Michigan, Michigan, USA
}

\begin{abstract}
This paper provides a new classification for morphing technology based on the functionality, operation, and the structural layout of the technology. In addition, it highlights the limitations of the conventional design approach to exploit the benefits of the technology using representative examples.

\section{INTRODUCTION}

There exist various overlapping definitions of morphing aircraft. According to Weisshaar [1], morphing is a technology or set of technologies that allows air-vehicles to alter their characteristics to achieve improved flight performance and control authority or to complete tasks that are not possible without this technology. The NATO RTO Technical Team on Morphing Vehicles suggested that morphing is the real-time adaptation to enable multi-point optimized performance [2]. A more detailed definition was provided by DARPA Morphing Aircraft Structures (MAS) program. According to Seigler [3], the MAS program defines the morphing aircraft as a multirole platform that changes its state substantially to adapt to changing mission environments, provides superior system capability not possible without reconfiguration, and uses a design that integrates innovative combinations of advanced materials, actuators, flow controllers, and mechanisms to achieve the state change.

The idea of morphing aircraft is not new. Even before the official beginning of controlled human flight in 1903, radical shape changing or morphing aircraft appeared and then disappeared, contributing little to aviation [1]. The reason for the disappearance of morphing was the increased need for larger structural rigidity as higher airspeeds were achieved which prohibited any form of compliance or flexibility or morphing. Later on, morphing mechanisms were added to improve the flight performance and control authority of the vehicle during off-design conditions. Recently, advances in materials, actuations, and multi-scale modelling allowed morphing aircraft to appear again and various research programs are focusing on the development of a full scale flying morphing aircraft.
\end{abstract}

\section{CATEGORISATION OF MORPHING AIRCRAFT}

Based on the definitions of morphing stressed in the previous sections flaps, slats, and retractable landing gears are all forms of adaptation or morphing that were adopted locally on conventional aircraft. The main reason for localised morphing is the need to improve operational performance or control authority of the aircraft without affecting the structural rigidity and integrity. Nowadays advances in materials and actuation systems facilitated structures with directional properties to allow flexibility while ensuring structural rigidity. The benefits of localised morphing have reached their upper threshold and only slight improvement is to be expected, which at one point means that discrete morphing cannot meet the continuous demand for more efficient and multi-mission aircraft.

\footnotetext{
${ }^{1}$ Lecturer in Aeronautics and Astronautics, University of Southampton, UK, Email: r.ajaj@southampton.ac.uk

${ }^{2}$ Research Associate,College of Engineering, Swansea University, UK, Email: c.s.beaverstock@swansea.ac.uk

${ }^{3}$ Professor in Aerospace Structures, Swansea University, UK, Email: m.i.friswell@swansea.ac.uk

${ }^{4}$ Professor, Head of Aeronautics \& Astronautics, University of Southampton, Email: andy.keane@soton.ac.uk

${ }^{5}$ Professor, Head of Aerospace Department, University of Michigan, MI, USA, Email: daninman@umich.edu
} 
Various categorisations of morphing were performed. Sofla et al. [4] and Barbarino et al. [5] categorised morphing based on geometric changes. These categorisations ignored conventional technologies such as flaps slats, landing gears, etc.... Therefore, categorisations based on geometric changes or mechanisms are not generic enough to handle all the forms of morphing. The authors believe that a more generic categorisation of morphing system is required based on the functionality, operational envelope, and application. Flaps, slats, and retractable landing gears are incorporated on conventional aircraft for singular functionality and they are only operated for a very short period of time along the flight envelope. In addition, they are only applied locally along the airframe, and they are designed not to carry the flight loads but to transform them to the airframe (wing or fuselage). These different systems are categorised as Discrete Morphing. Discrete morphing can be regarded as a very mature technology as it has been used for almost 100 years. Therefore only slight benefits are to be expected and their abilities to assist in meeting the future stringent requirements are doubtable. The ultimate objective for the future aircraft is what so called Continuous Morphing where one system can provide multiple functionalities in a continuous fashion along the flight envelope, and these systems are capable of carrying the various flight loads. Table 1 summarises the definitions and differences between Discrete and Continuous Morphing.

Table 1: Definitions of Discrete and Continuous Morphing

\begin{tabular}{lll}
\hline \hline \multicolumn{1}{c}{ Discrete Morphing } & \multicolumn{1}{c}{ Continuous Morphing } \\
\hline - Singular functionality. & Multiple functionalities. \\
- Adopted locally on board the aircraft & - Adopted all over the body of the aircraft. \\
- Operated at few points of the flight & - Operated continuously along the flight \\
envelope. & $\begin{array}{l}\text { envelope. } \\
\text { Suppress coupling between the aircraft } \\
\text { axes. }\end{array}$ & $\begin{array}{l}\text { Exploit couplings in morphing schedules } \\
\text { and between the aircraft axes. }\end{array}$ \\
\hline
\end{tabular}

Continuous morphing can be seen mainly in nature through birds' wings that can perform various missions (loiter and strike) and different functionalities (control and flight performance). Examples of both Discrete and Continuous Morphing are shown in Figure 1.

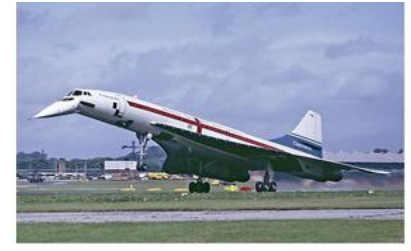

Variable incidence nose

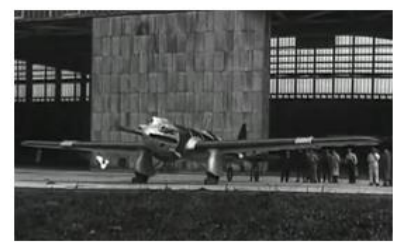

Variable span wing

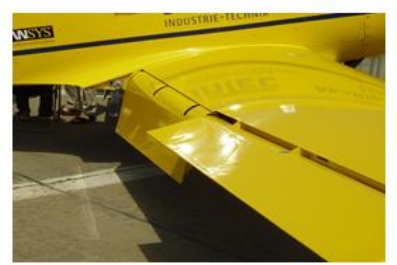

Flaps

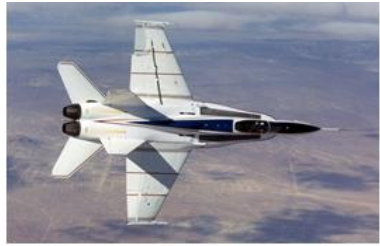

Active Aeroelastic Wing

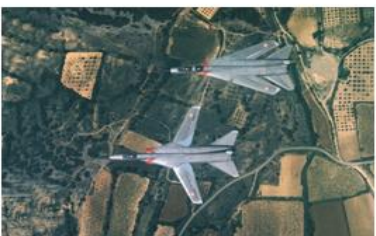

Variable sweep wing

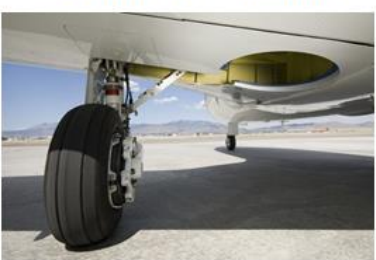

Retractable landing gear

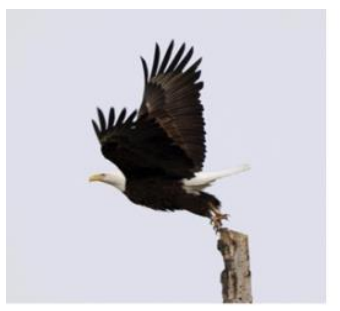

Take-off

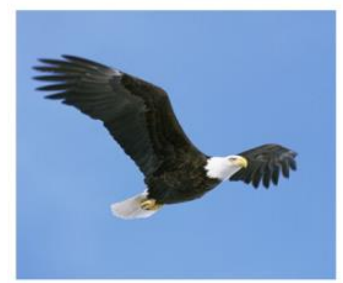

Strike

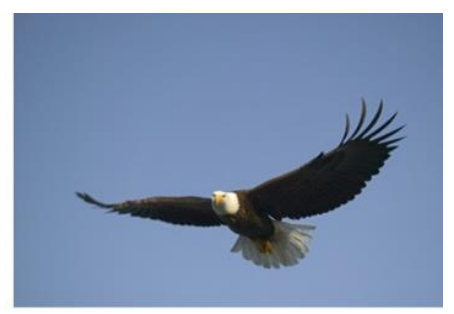

Loiter

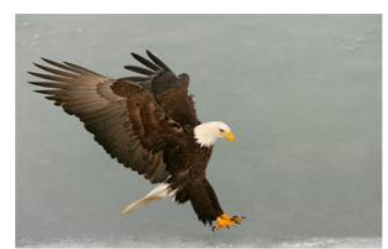

Landing

a. Discrete Morphing

b. Continuous Morphing

Figure 1: Examples of Discrete and Continuous Morphing

\section{MORPHING AIRCRAFT DESIGN}

Most of today's aircraft are designed according to Cayley's design paradigm which separates the functions needed for sustained flight [6]. During conventional conceptual design, the geometry of conventional aircraft is usually optimised for a single flight segment (cruise for transport aircraft), which 
results in a penalised behaviour at off-design conditions [7]. To overcome the penalty in off-design conditions, discrete surfaces such as flaps and slats are added to allow changes in the wing profile and have better flight characteristics at off-design conditions. On the contrary, morphing aircraft can alter their geometry according to the instantaneous flight conditions to have the optimum flight characteristics depending on the mission objectives. The ability to adapt their geometries infers that morphing vehicles can perform multiple missions.

\section{Multi-Axis Effectors}

Most of the studies in literature have focused on using morphing systems to replace conventional systems. However, conventional systems are more than 100 years old and the way they are operated have been exploited and optimised over time. The way conventional systems are operated depends on the system itself and its technology. Therefore, operating morphing systems that is associated with different form of technology in the same way as conventional systems might constrain the benefits of morphing systems and results in them being inferior candidates.

To illustrate, consider a roll manoeuvre for a medium altitude long endurance (MALE) UAV. A comparison between conventional ailerons and asymmetric span morphing is performed. The conventional ailerons are deflected asymmetrically, and the rolling moment generated is a function of the ailerons size, spanwise position of the aileron, deflection angle, and the dynamic pressure. The rolling moment generated by ailerons is independent of the angle of attack (assuming linear aerodynamic regime). For asymmetric span morphing, the rolling moment generated is dependent on the change in wing span, dynamic pressure, and the instantaneous angle of attack. Figure 2 shows the variation of rolling moment with the AOA for both ailerons and span morphing at the same dynamic pressure.

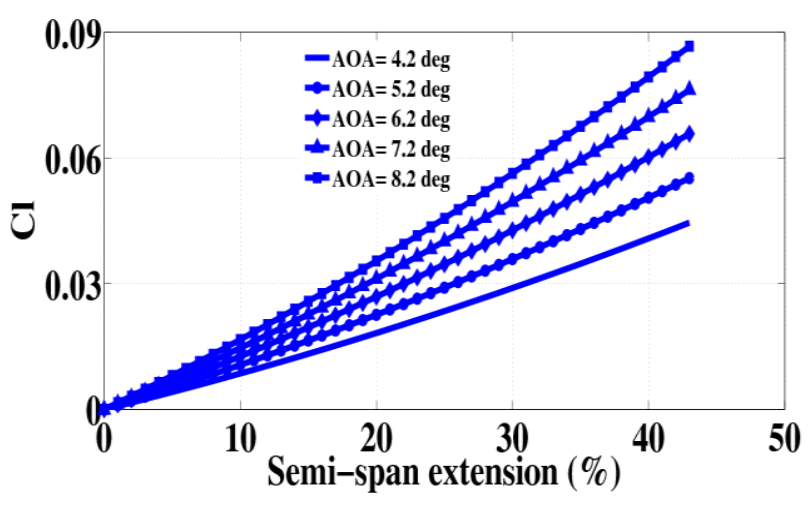

a. With span

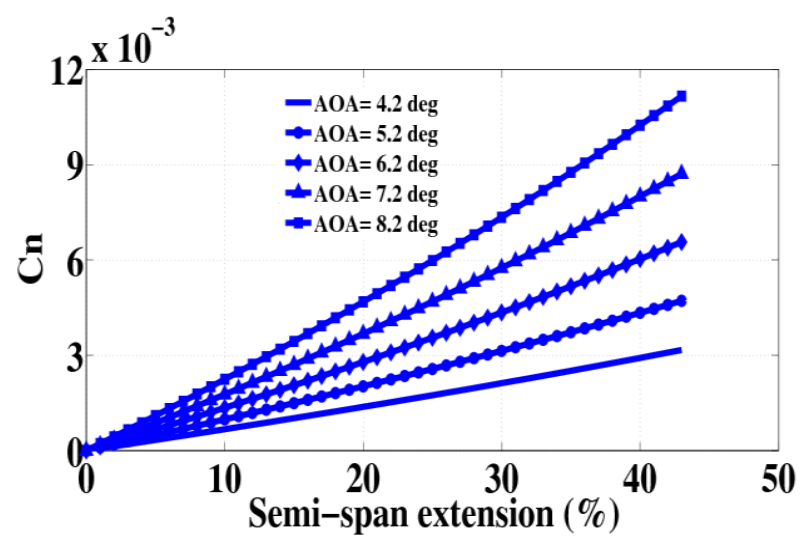

c. With span

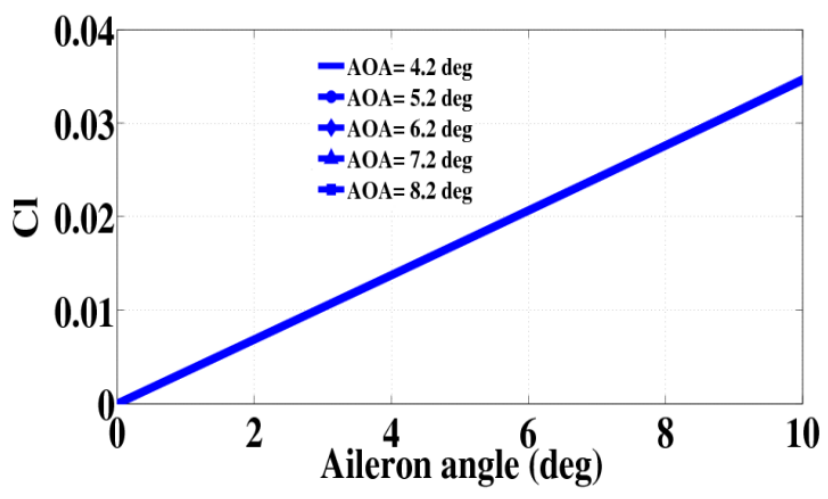

b. With aileron

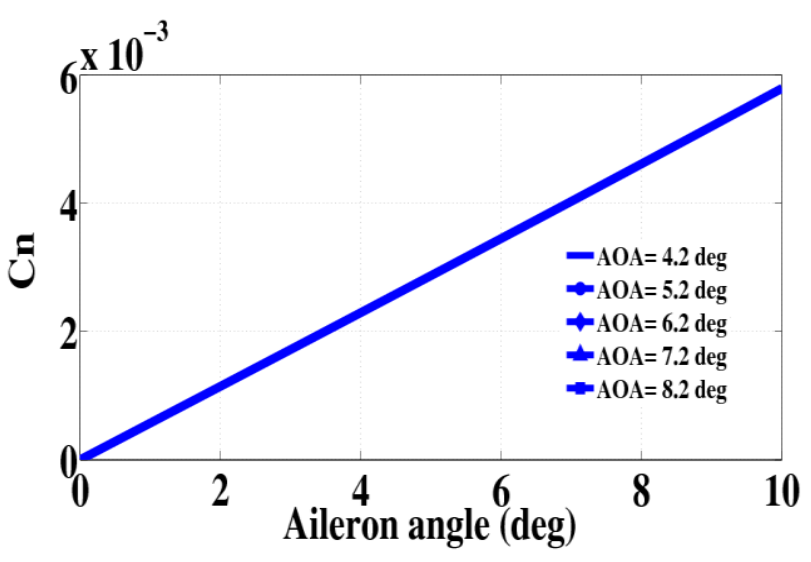

d. With aileron

Figure 2: Variation of rolling and yawing moment coefficients from span morphing and aileron angle with the angle of attack at M0.16, 20,000 ft

It should be noted that the wing of the UAV is unswept and hence asymmetric span extension or retraction only affect roll and yaw (and not pitch). By examining Figure, one can notice the great sensitivity of rolling moment from span morphing to the angle of attack which doesn't exist for conventional ailerons. If this sensitivity to the is neglected, then from a structural and actuation point of 
view, conventional ailerons are a superior choice to provide roll control, due to the lighter weight, lower actuation power, and minimal complexity. However, if the coupling between pitch and roll is considered, then span morphing can be a superior option due to the large level of manoeuvrability that can be achieved for a wide range of flight conditions.

Similarly, Bourdin et al. [8] demonstrated that significantly coupling effects can be achieved with the morphing winglet (variable cant angle) when actuated asymmetrically on board of a flying wing as shown in Figure 3. The large coupling between roll, yaw, and pitch allowed coordinated turns and increased the agility of the vehicle.
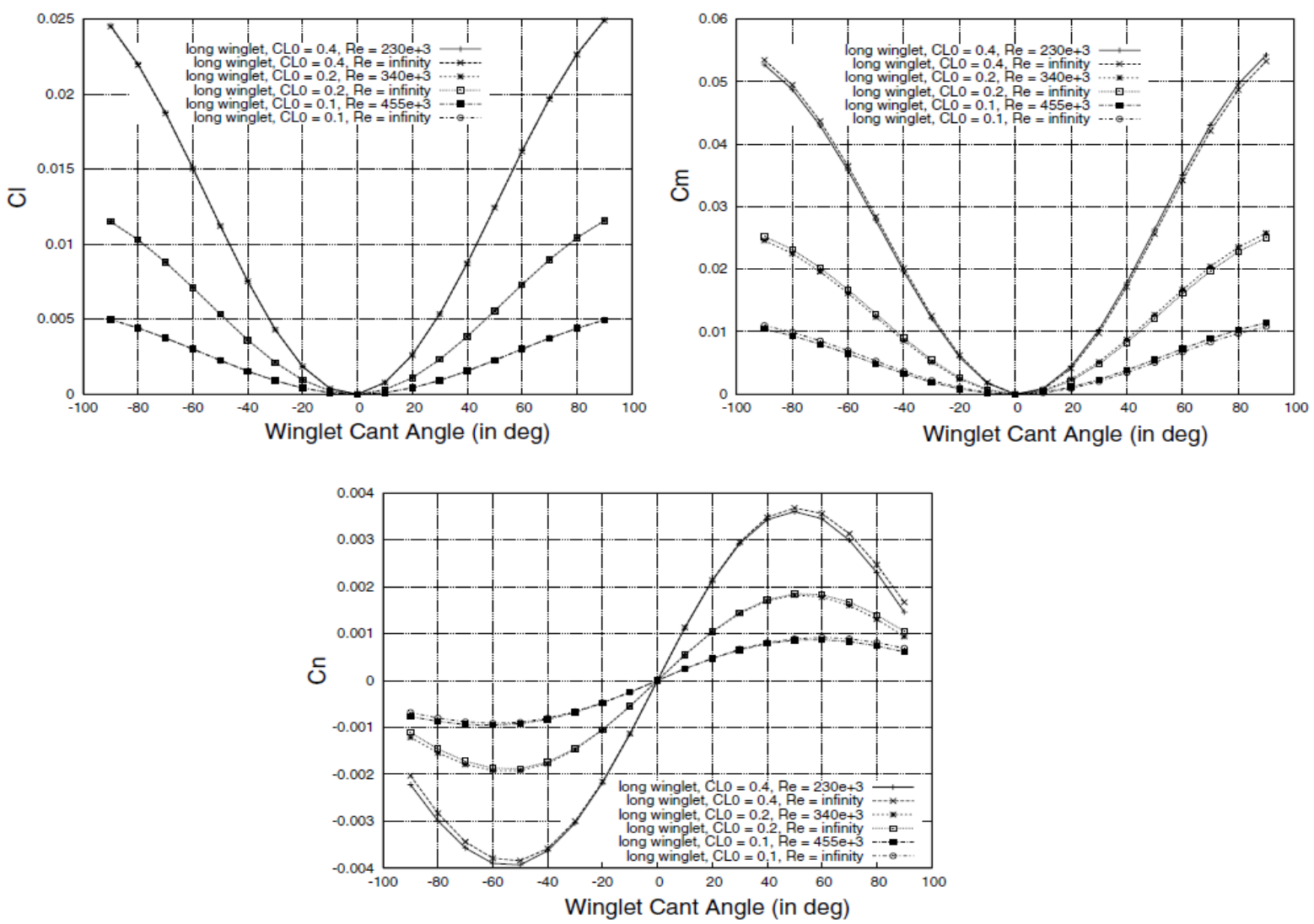

Figure 3: Moments attainable by folding up or down the right winglet while the left winglet remains planar [8]

The conventional design approach has been to design systems with minimal inherit coupling to simply the design of the control law and improve handling/riding quality. However, recent advances in computers and the continuous expansion of the UAV and RC aircraft market offers a good opportunity to exploit those coupling with morphing systems to enhance the agility or the operational performance of the aircraft.

\section{Functionality}

Conventional systems are usually designed to perform a single functionality. Slats and flaps are a good example because they are only used to reduce the stall speed during take-off and landing. This single functionality of those massive systems limits their use for a very short period of time of the entire mission. This single functionality design approach has been mainly used for safety and redundancy factors which resulted in a large number of systems with reduced number of functionalities. Most of the studies in literature, compares between the functionality of a morphing system with the functionality of a conventional system. This kind of studies cannot fully address the benefits of morphing due to the one-to-one comparison. This constrained the benefits that can be possibly achieved with morphing.

Morphing systems must be designed to have multiple functionalities and not only to have the functionality of conventional systems that they are aimed to replace. For instance, asymmetric span morphing (discussed in Section A) that is used for roll control can be used to enhance aerodynamic efficiency (drag reduction) during steady flight phases and enhance operational performance during transitional flight phases (reduce take-off and landing distances) when actuated symmetrically. As stressed before, if only the roll control functionality of span morphing is considered, then ailerons are a more effective roll device in terms of weight, actuation, complexity, and knowledge. However, ailerons 
can't enhance aerodynamic efficiency or operational performance, and in order to enhance aerodynamic efficiency or operational performance, other conventional systems are required which increases overall weight and reduces efficiency. Figure 4 shows the improvement in endurance and the reductions in TOFL and LD that can be achieved with symmetric span morphing (extension).

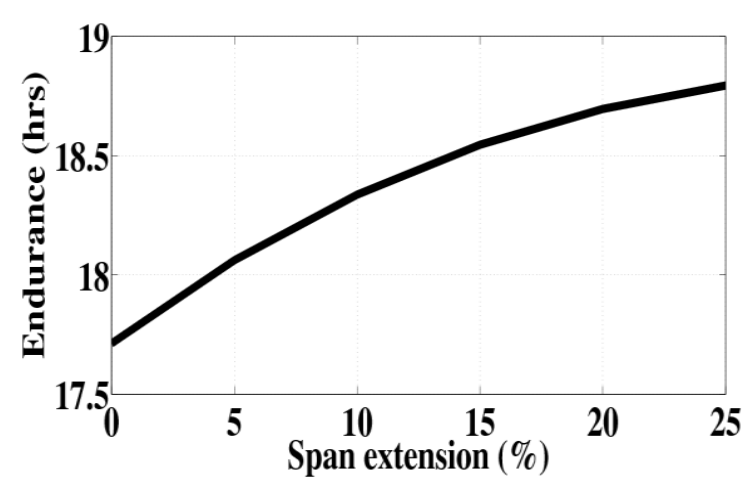

a. Endurance

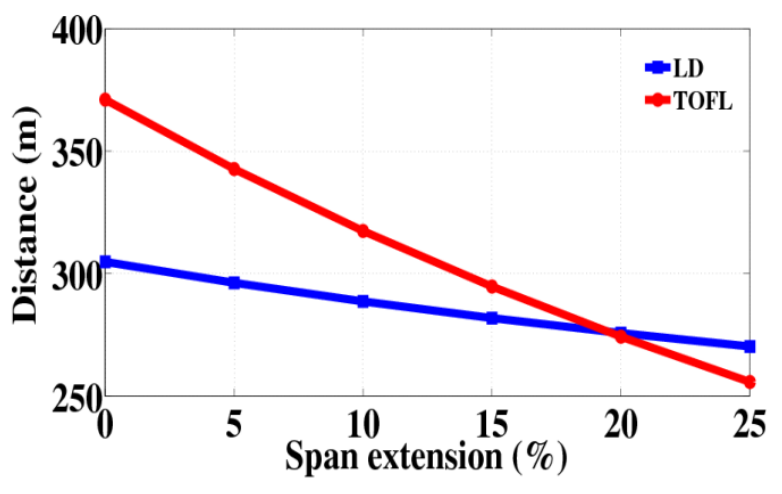

b. TOFL and LD

Figure 4: Operational performance improvements with symmetric span morphing

In addition, symmetric span retraction allows the UAV to be more agile to perform a dash flight. Similarly, Smith et al. [9] used the morphing winglet symmetrically on the wingtip of a commercial transport aircraft similar to the Airbus A320-200. They quoted about $4 \%$ improvements in specific air range (SAR) during the mid and final cruise flight segments.

With the multi-functionality design approach for morphing technologies figures of merits (FOMs) such as power or weight become misrepresentative. Therefore a new set of FOMs have to be developed to allow a fair comparison between conventional and morphing systems. For instance, instead of using weight or power as the FOM, weight per functionality or power per functionality can be used. If these new FOMs are not introduced, then it will be impractical to have a good assessment of the benefits of morphing.

\section{Compliance vs. Mechanisms}

Another important factor in morphing systems is the scale of the air-vehicle on which they will be incorporated. All the morphing concepts available in literature can be categorised to be either compliant, mechanisms, or hybrid (mixture of compliant and mechanisms). Compliant structures are promising solutions due their low weight and maintenance costs (no rigid body motion). Compliant structures usually employ flexible skins to maintain the aerodynamic shape of the wing while before, during, and after morphing. There is a wide range of flexible skins ranging from corrugated skins to fibre reinforced elastomer. Thill et al. [10] provided an extensive review of the state-of-the-art morphing skins. The drawback of the state-of-the-art flexible skins is that they can't work as main load carrier members. Their main purpose is to maintain the aerodynamic profile of the wing and transfer the pressure loads to the inner main load carrier structures. Compliant structures seem to work well for small UAVs and RC aircraft. In addition, they can be used in wind turbine blades of various sizes due to the relatively lower dynamic pressure. However, as the size/weight of the vehicle increase and hence the aerodynamic loads, it becomes prohibitive to employ compliant structures due to their relatively low stiffness and strength.

Consider 2 UAVs, the Tekever AR4 and the BAE Systems Herti. The UAVs have different wing areas and MTOWs as listed in Table 2. Assume that elastomeric skins are used on the wing of each UAV. The width of the skin plate is equal to the wing chord and its length is equal to the rib spacing of $0.35 \mathrm{~m}$. The skin is modelled as a simply supported plate on the 4 sides.

Table 2: Scalability of compliant structures

\begin{tabular}{ccc}
\hline \hline Parameters & AR4 & Herti \\
\hline MTOW $(\mathrm{kg})$ & 5 & 800 \\
Wing loading $\left(\mathrm{kg} / \mathrm{m}^{2}\right)$ & 12 & 36 \\
$c(\mathrm{~m})$ & 0.24 & 1.87 \\
\hline
\end{tabular}

Under normal aerodynamic loading, the maximum allowed deformation of the skin is $0.005 \mathrm{~m}$. The thicknesses, axial stiffness and weight of the two skins are summarised in Table 3. 
Table 3: Scalability Analysis

\begin{tabular}{ccc}
\hline \hline Parameters & AR4 & Herti \\
\hline$t(\mathrm{~mm})$ & 9 & 34 \\
$K_{x}\left(\times 10^{6} \mathrm{~N} / \mathrm{m}\right)$ & 0.24 & 8.8 \\
$W(\mathrm{~kg})$ & 0.7 & 1.9 \\
\hline
\end{tabular}

The analysis above assumes the elastomeric skin to have a Young's modulus of $0.05 \mathrm{GPa}$ and a mass density of $900 \mathrm{~kg} / \mathrm{m}^{3}$. It should be noted that the skin thickness for the Herti is 3.8 that of the AR4, while the axial stiffness of the Herti's skin is 37 times that of the AR4's skin. This implies that the actuation force needed to morph the Herti's skin is 37 times that of the AR4.

\section{Design with Morphing}

One drawback with the studies on morphing aircraft is that morphing is added to an existing aircraft. This retro-fitting approach directly eliminates some major benefits of the morphing technology and constrains any potential for multi-functionality. With morphing systems, the structure is strongly coupled with the actuator(s) and with the sensor(s) and in some morphing systems the structure is the actuator and is the sensor at the same time. This coupling doesn't exist in conventional systems. Therefore adding morphing to an existing aircraft generates different outcomes (in terms of benefits) to considering morphing early in the design process of the aircraft. This will be addressed further in future work.

\section{CONCLUSIONS}

The comparison between morphing and conventional aircraft requires new figures of merit that can account for the multifunctional and scale-dependent natures of morphing. This implies that the conceptual design approach has to be modified to allow for morphing to be parameterised and optimised early in the design process rather than being retrofitted. In future work, the integration of the morphing parameters with the conventional conceptual design will be performed. In addition representative examples for the conceptual design of morphing aircraft will be included.

\section{ACKNOWLEDGMENTS}

Michael Friswell acknowledges receiving funding from the European Research Council under the European Union's Seventh Framework Programme (FP/2007-2013) / ERC Grant Agreement n. [247045].

\section{References}

Weisshaar, T.A. (2006). Morphing Aircraft Technology-New Shapes for Aircraft Design, RTO-MP-AVT141, Neuilly-sur-Seine, France.

McGowan, A.R., Vicroy, D.D., Busan, R.C. and Hahn, A.S. (2009). Perspectives on Highly Adaptable or Morphing Aircraft, RTO Applied Vehicle Technology Panel (AVT) Symposium, Evora, Portugal, 20-24 April 2009, RTO-MP-AVT-168 AC/323(AVT-168)TP/268.

Seigler, T.M. (2005). Dynamics and Control of Morphing Aircraft, PhD Thesis, Virginia Polytechnic Institute and State University.

Sofla, A.Y.N., Meguid, S.A., Tan, K.T. and Yeo, W.K. (2010). Shape Morphing of Aircraft Wing: Status and Challenges, Materials and Design, 31:1284-1292.

Barbarino, S., Bilgen, O., Ajaj, R.M., Friswell, M.I., and Inman, D.J. (2011). A Review of Morphing Aircraft, Journal of Intelligent Materials and Smart Structures, 22(9):823-877.

Melin, T. (2006). Multidisciplinary Design in Aeronautics, Enhanced by Simulation- Experiment Synergy, PhD Thesis, Royal Institute of Technology (KTH), Sweden, 2006, Report: TRITA AVE 2006:25.

Raymer, D.(2006). Aircraft Design: A Conceptual Approach, American Institute of Aeronautics and Astronautics (AIAA), 4th Edition.

Bourdin, P., Gatto, A., and Friswell, M.I. (2008). Aircraft Control via Variable Cant-Angle Winglets, Journal of Aircraft, Vol.45, No.2, March-April.

C. Thill, J. A. Etches, I. P. Bond, K. D. Potter, and P. M. Weaver. (2008). Morphing Skins - A Review, The Aeronautical Journal 112, pp. 117-139, 2008. 
Smith, D.D., Ajaj, R.M., Isikveren, A.T., and Friswell, M.I. (2012). Multi-Objective Optimization for the Multiphase Design of Active Polymorphing Wings, Journal of Aircraft, 49(4), July-August 2012, 1153-1160. 